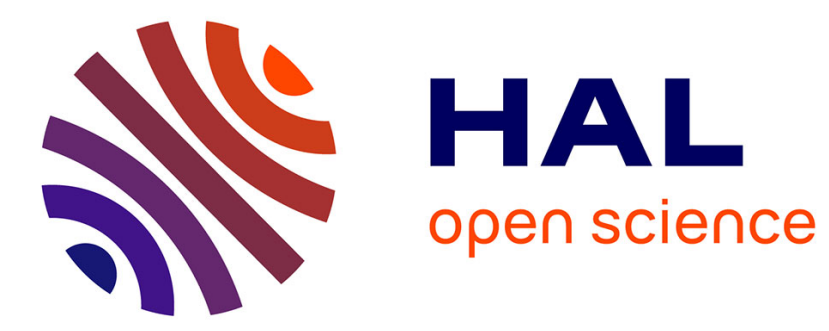

\title{
La notion de chaos en science et dans la Bible.
}

Jacques Fantino

\section{To cite this version:}

Jacques Fantino. La notion de chaos en science et dans la Bible.. Revue des Sciences Religieuses, 2000, 74, pp.292-303. 10.3406/rscir.2000.3538 . hal-01389790

\section{HAL Id: hal-01389790 \\ https://hal.science/hal-01389790}

Submitted on 29 Oct 2016

HAL is a multi-disciplinary open access archive for the deposit and dissemination of scientific research documents, whether they are published or not. The documents may come from teaching and research institutions in France or abroad, or from public or private research centers.
L'archive ouverte pluridisciplinaire HAL, est destinée au dépôt et à la diffusion de documents scientifiques de niveau recherche, publiés ou non, émanant des établissements d'enseignement et de recherche français ou étrangers, des laboratoires publics ou privés. 


\section{La notion de chaos en science et dans la Bible} Jacques Fantino

\section{Citer ce document / Cite this document :}

Fantino Jacques. La notion de chaos en science et dans la Bible. In: Revue des Sciences Religieuses, tome 74, fascicule 3, 2000. pp. 292-303;

doi : 10.3406/rscir.2000.3538

http://www.persee.fr/doc/rscir_0035-2217_2000_num_74_3_3538

Document généré le 03/06/2016 


\begin{abstract}
Science has first stutied phenomena which are regular, stable, and recurrent. Now science is also interested in the order and the disorder appearing in physical systems. This the reason why science analyzes the structuration of the physical systems and their evolution during time. A lot of results have been found out, throwing a light upon the processes which causes order or disorder in physical systems. The first part of this paper shows some results about chaos in physical systems.

In order to bring into relation the scientific view of chaos which the Christian doctrine of creation, the second part introduces what the Bible tells about order and disorder in creation. Some texts are produced in order to give the biblical notion of chaos. Scientific interpretation of chaos is then compared to the biblical teaching : what science and theology say about the world and the chaos in it. More radically, the question is to know if chaos has the same meaning in science and in the Bible (and thus in theology).
\end{abstract}

\title{
Résumé
}

Les sciences ont d'abord recherché les lois qui gouvernent les phénomènes réguliers, stables ou répétitifs. Puis, ces dernières décennies elles se sont intéressées à l'ordre et au désordre qui se manifestent dans les systèmes physiques. Ainsi, en étudiant la structure des systèmes et leur évolution dans le temps, les sciences ont mis en évidence les processus qui conduisent à l'apparition de l'ordre ou du désordre dans les systèmes. La communication présente quelques-uns des résultats concernant le chaos dans les systèmes physiques.

Afin de mettre en relation cet enseignement avec la théologie de la création, on présente dans une deuxième partie ce que la Bible dit de la présence d'ordre et de désordre dans le créé. La communication interprète le donné biblique à partir de quelques textes. L'image produite par l'interprétation scientifique de la nature peut alors être comparée avec celle qui résulte de l'interprétation théologique du monde comme création. Cette comparaison permet d'examiner ce que science et théologie disent de la nature et de la fonction du chaos dans le monde et, plus radicalement, si les notions de chaos correspondent aux mêmes réalités en science et en théologie. 
Revue des sciences religieuses $74 \mathrm{n}^{\circ} 3$ (2000), p. 292-303

\section{LA NOTION DE CHAOS EN SCIENCE ET DANS LA BIBLE}

La théologie chrétienne s'est toujours construite en fonction de la culture dans laquelle vivent les croyants. La théologie interprète en effet la révélation selon la compréhension du monde appartenant à cette culture (1). Ce constat vaut pour la théologie en général, mais il l'est d'une manière plus nette encore pour la théologie de la création. La formulation de la révélation chrétienne concernant le monde comme créé passe par la compréhension profane du monde.

Or, le développement scientifique des deux derniers siècles a complètement transformé l'image de la nature et de la vie. L'étude des phénomènes conduisant à l'apparition de l'ordre ou du désordre, voire du chaos, dans les réalités physiques constitue un exemple récent du renouveau de l'approche de la nature. Est-ce que cela change quelque chose au discours théologique sur la création? En effet, comme le fait remarquer A. Ganoczy, si la tradition théologique a valorisé la notion d'ordre pour parler du créé, elle a laissé en arrière plan celle de chaos, souvent en la dépréciant (2).

Parler ou reparler du chaos en théologie sous l'impulsion des nouveaux acquis scientifiques suppose cependant une première étape méthodologique. Elle consiste à clarifier le sens et l'usage de la notion de chaos tant en science qu'en théologie de la création. Préciser de quoi il est question dans chacune des deux disciplines permet en un second temps de voir l'éclairage que chacune peut éventuellement apporter à l'autre. L'étude qui suit est une contribution à cette première étape.

(1) Cette manière de faire est déjà à l'œuvre dans les Écritures; cf. J. Barr, Biblical Faith and Natural Theology, Oxford, Clarendon Press, 1994.

(2) Voir A. Ganoczy, Chaos - Zufall - Schöpfungsglaube, Mainz, M. Grünewald Verlag, 1995, p. 132. 


\section{Le chaos selon la théorie des systèmes dynamiques}

Le problème de l'évolution à long terme des systèmes physiques est né des recherches menées au XIX ${ }^{\mathrm{e}}$ siècle sur la stabilité du système solaire. Pour cela on rapporte le mouvement des planètes à un référentiel lié au soleil, considéré alors comme fixe. Les variables de ce système particulier sont les positions et les vitesses des planètes et de leurs satellites. La question de la stabilité peut être posée par exemple en se demandant si une planète, ou un satellite, peut «s'évader " du système solaire. Ou encore si les planètes tournent autour du soleil selon un mouvement suffisamment régulier pour pouvoir durer indéfiniment, sauf perturbation due à un élément extérieur au système solaire ou à l'évolution interne de l'étoile qu'est le soleil. Pour tenter de répondre à ces questions et savoir si le système solaire est stable, il faut résoudre les équations décrivant le mouvement des différents corps constituant le système solaire. On savait au siècle dernier construire des solutions approchées de ces équations, mais on n'avait pas déterminé, au moins théoriquement, leurs solutions exactes. À ce sujet $\mathrm{H}$. Poincaré a montré deux résultats essentiels (3). D'une part, il est impossible de résoudre ces équations de manière analytique, on ne peut construire que des solutions approchées. D'autre part, à long terme ces solutions ne valent plus. La nature même du système solaire fait qu'on ne peut pas approcher son évolution à long terme. Dit autrement, la position des planètes n'est pas prédictible au-delà d'une plage de temps déterminée. Inversement, on ne peut pas non plus connaître leur position dans le passé au-delà d'un intervalle de temps identique (4). Ces résultats sont généralisables à tous les systèmes et les travaux de Poincaré sont au fondement de la théorie des systèmes dynamiques, branche de la physique qui étudie les systèmes dans leur évolution.

Dans les limites imparties à cette étude nous considérons l'évolution des systèmes physiques. Tout système est caractérisé par des variables déterminant l'évolution du système dans son environnement. Si l'on étudie par exemple l'écoulement d'un fluide dans un canal, on prend comme variables la masse volumique, la pression et le débit en chaque point de la section du canal. L'intérêt est de déterminer l'écoulement du fluide dans le canal, pas le mouvement des molécules en tant que tel.

Tout système physique est ainsi fonction des variables qui le caractérisent et son évolution est régie par les lois physiques gouver-

(3) Voir F. Lurçat, Le chaos, Paris, PUF, 1999, p. 55-70.

(4) Cette plage de temps est d'environ 10 millions d'années. À titre de comparaison rappelons que l'âge du système solaire est de l'ordre de 4,5 milliards d'années. 
nant la dynamique du système. Mathématiquement, l'évolution est une solution d'un ensemble d'équations qui ne sont pas linéaires, d'où l'appellation de systèmes non linéaires (5).

On s'intéresse tout d'abord à un système soumis à des contraintes données et supposées pour l'instant constantes. Dans le cas général on montre que dans tel un environnement stable et pour des valeurs bornées des variables, le régime d'un système quelconque tend à long terme vers un régime particulier, stationnaire, périodique ou quasipériodique.

Les régimes stationnaires constituent les états d'équilibre du système (6). L'écoulement lent de l'eau dans un canal en est un bon exemple. Il n'y a pas de turbulence.

Les régimes périodiques sont constitués par les solutions périodiques des équations. Dans la pratique beaucoup de systèmes sont en effet le siège de phénomènes périodiques. C'est le cas par exemple de nombreuses réactions chimiques, notamment dans le métabolisme de la cellule (7).

Les régimes quasipériodiques résultent de la superposition d'un nombre fini de solutions périodiques dont les périodes ne sont pas commensurables (c'est-à-dire des multiples entiers l'une de l'autre). Un bon exemple est le système solaire où chaque planète a un régime de ce genre. Ainsi la Terre est soumise à l'action du soleil, de la lune et des autres planètes et de leurs satellites. Le mouvement résultant est la superposition des ces différents mouvements périodiques.

Dire que la dynamique d'un système quelconque tend vers un régime permanent, c'est affirmer qu'il y est attiré. On rencontre ainsi la notion d'attracteur (8). Un attracteur est un de ces régimes particuliers, stationnaire, périodique ou quasipériodique, vers lequel tend tout régime du système, pourvu que les valeurs des variables du

(5) $\grave{A}$ propos des systèmes dynamiques, voir, outre l'ouvrage introductif de F. Lurçat cité auparavant, P. Manneville, «Structures dissipatives et turbulence », in S. Aubry et alii (éd.), Structures et instabilités, Les Ulis, Les Éditions de Physique, 1986 ; P. Bergé, Y. Pomeau, C. Vidal, L'ordre dans le chaos. Vers une approche déterministe de la turbulence, Paris, Hermann, $1988^{2}$; F. Verhulst, Non linear Differential Equations and Dynamical Systems, Heidelberg, Springer-Verlag, 1990 ; L. Perko, Differential Equations and Dynamical Systems (Texts in Applied Mathematics, 7), New York, Springer-Verlag, 1991 ; A. Lesne, Méthodes de renormalisation. Phénomènes critiques, chaos, structures fractales, Paris, Eyrolles, 1996.

(6) Cf. Bergé, Pomeau, Vidal, L'ordre dans le chaos, p. 293 ; Verhulst, Nonlinear Differential Equations and Dynamical Systems, p. 11.

(7) Cf. Bergé, Pomeau, Vidal, L'ordre dans le chaos, p. 3-9.

(8) Cf. Manneville, "Structures dissipatives et turbulence ", p. 356-357 ; Lesne. Méthodes de renormalisation, p. 150-151. 
système restent dans un même domaine (9). Ce domaine constitue la zone d'attraction.

Un attracteur peut correspondre à un régime stable. C'est le cas par exemple de tout appareil que l'on met en marche : moteur, appareil électronique... Après un temps de transition, l'appareil atteint son régime permanent. Mais l'attracteur peut aussi bien être instable. Cela signifie alors une grande sensibilité aux conditions initiales, c'està-dire aux valeurs que les variables avaient quand l'étude du système a commencé.

Vue son importance, explicitons la notion de stabilité d'un régime correspondant à un attracteur. Un régime est stable si une modification des conditions initiales n'entraîne pas de changement qualitatif notoire du comportement du système. Plus précisément, considérons un système fonctionnant selon un certain régime atteint à partir de certaines conditions initiales, c'est-à-dire de certaines valeurs des variables du système à sa mise en route. Si nous prenons le même système, ou un autre qui lui soit identique, et qu'on le mette en route avec des conditions initiales légèrement différentes, le régime de fonctionnement atteint sera du même type que celui du système précédent avec des valeurs légèrement différentes de fonctionnement. Ceci si le premier régime est stable. S'il ne l'est pas, alors le fait de modifier légèrement les conditions initiales à la mise en route conduit à un régime de fonctionnement assez différent. En définitive, la stabilité ou l'instabilité du régime de fonctionnement d'un système se vérifie en modifiant un peu ses conditions initiales et en observant le nouveau régime de fonctionnement atteint. Il y a stabilité quand le régime atteint après la modification est différent mais voisin qualitativement et quantitativement du premier.

Pour un attracteur instable en revanche une légère modification des conditions initiales entraîne le passage à un régime très différent. On voit que l'instabilité est liée à la sensibilité du système aux conditions initiales. On passe dans le cas d'un attracteur stable à un régime voisin de celui de départ, tandis que pour un attracteur instable on atteint un régime éloigné du premier. Ce phénomène est encore renforcé si l'environnement est variable.

Nous avons effectivement envisagé jusqu'ici le comportement d'un système laissé à lui-même dans un environnement constant. Ce cas n'est qu'une approximation. Les changements de l'environnement

(9) Cf. Bergé, Pomeau, Vidal, L'ordre dans le chaos, p. 108 et 117-118; Verhulst, Nonlinear Differential Equations and Dynamical Systems, p. 12 ; L. Perko, Differential Equations and Dynamical Systems, p. 177. 
influent encore plus sur le régime de fonctionnement du système. En effet, les variables caractérisant le système, ou au moins certaines d'entre elles, dépendent de facteurs largement conditionnés par le milieu extérieur, comme la température, la composition chimique, etc. Le régime de fonctionnement du système n'est alors plus seulement fonction des variables qui le caractérisent mais aussi de ces facteurs extérieurs. Ce sont ces facteurs, encore appelés paramètres qui vont avoir une influence déterminante sur l'évolution du système. Reprenant l'exemple de l'écoulement d'un fluide dans un canal, un paramètre important sera la température. Comme l'expérience courante le prouve, un fluide s'écoule d'autant mieux en général que sa température est plus élevée parce que sa viscosité diminue avec la température. On voit sur cet exemple que la modification d'un paramètre modifie les conditions de fonctionnement d'un système et éventuellement peut en changer le régime.

Ainsi, à toute valeur d'un paramètre correspond une solution du système d'équations représentant l'évolution du système. Toute modification, même minime, d'un paramètre a pour conséquence de faire passer d'une solution à une autre, d'un régime dynamique à un autre. Autrement dit, la considération des seules variables caractérisant le système conduit à un seul régime possible correspondant à une solution unique des équations. Mais, la prise en compte de contraintes variables de l'environnement, sous la forme de paramètres, fait apparaître une multiplicité de solutions possibles là où il n'y en avait qu'une seule.

En général, le régime du système résultant d'une variation légère d'un paramètre caractérisant l'action de l'environnement sur le système est voisin de celui qu'il avait auparavant. Cependant, quand un paramètre atteint certaines valeurs qualifiées de critiques, plusieurs solutions sont possibles en même temps correspondant chacune à un régime particulier du système. Bien sûr le système se trouvera dans l'un de ces régimes seulement. Le passage d'une solution unique à plusieurs solutions possibles, à cause de la variation d'un paramètre, s'appelle une bifurcation (10). La modification d'un paramètre peut ainsi entraîner des changements qualitatifs importants. Le régime d'un système change alors de nature ou de stabilité, ce qui est précisément le cas lors d'une bifurcation (11). Tel est le cas de l'amplificateur d'une chaîne Hi-Fi qui, à cause de l'évolution de certains de ses composants électroniques, se met à siffler, c'est-à-dire à osciller. Le

(10) Cf. Bergé, Pomeau, Vidal, L'ordre dans le chaos, p. 293 ; M. Demazure, Catastrophes et bifurcations, Paris, Ellipses, 1989, p. 121-122 et 297 s. ; Verhulst, Nonlinear Differential Equations and Dynamical Systems, p. 183-185

(11) Cf. Manneville, "Structures dissipatives et turbulence ", p. 342. 
régime du système, ici l'amplificateur, a qualitativement changé, puisqu'il est devenu un oscillateur.

Les bifurcations dépendent de la variation de paramètres, qui en général traduisent l'impact de l'environnement sur le système. Ce sont donc bien les conditions variables de l'environnement qui renforcent les possibilité de changement dans les régimes dynamiques des systèmes.

La notion de bifurcation permet de comprendre le passage à un chaos que l'on dit déterministe. Un système, du fait de la variation d'un paramc̀trc, peut subir un enchaînement de bifurcations qui entrấnent le système dans un régime erratique totalement déterminé par cette série d'évolutions, d'où le nom de chaos déterministe. Le système est alors entraîné par des processus internes de plus en plus erratiques dans une évolution irréversible vers le chaos.

Le passage à un régime chaotique est un cas de figure extrême qui peut même aboutir à la destruction du système. En général, par la variation d'un paramètre, le système passe d'un régime permanent à un régime voisin. Quand tel est le cas, on dit que le système est stable par rapport à la variation du paramètre en question. Ce résultat ne vaut évidemment que pour une plage de variation limitée du paramètre considéré. Au-delà, le système atteint une bifurcation. Il peut alors passer à un autre régime permanent, qualitativement différend du premier. C'est le cas d'un amplificateur qui se met à " accrocher » ou à « siffler ", c'est-à-dire à osciller. Ou bien le système évolue vers un régime chaotique.

La théorie des systèmes dynamiques met en évidence des scénarios génériques universels qui expliquent comment par la variation d'un paramètre d'un système donné celui-ci passe d'un régime permanent à un régime chaotique (12).

La sensibilité aux conditions initiales est caractéristique d'une dynamique évoluant vers le chaos. Pour illustrer cet aspect, considérons deux systèmes identiques, mais avec des conditions initiales légèrement différentes. Les évolutions respectives des deux systèmes vont très rapidement apparaître divergentes. Ce phénomène est dû à l'amplification imprédictible des différences, caractéristique propre aux évolutions chaotiques. Sensibilité aux conditions initiales et existence d'un attracteur sont tous deux nécessaires pour passer au chaos, l'attracteur ne suffit pas à lui seul (13). Mais, dans ce cas l'attracteur

(12) Cf. Lesne, Méthodes de renormalisation, p. 159.

(13) Cf. Manneville, "Structures dissipatives et turbulence », p. 385 ; Bergé, Pomeau, Vidal, L ordre dans le chaos, p. 176 ; Lesne, Méthodes de renormalisation, p. $155-157$. 
est appelé étrange à cause précisément du comportement chaotique du système.

La tendance au chaos se rencontre pour tout système physique. C'est le cas des systèmes dissipatifs qui possèdent tous au moins un attracteur. On a vu que beaucoup de systèmes, la plupart même, possèdent comme régimes permanents des régimes périodiques ou quasipériodiques, superposition d'un nombre fini de solutions périodiques appelées modes. Une bifurcation est engendrée par la perte de stabilité d'un régime périodique ou d'un mode d'un régime quasipériodique. Plusieurs scénarios sont alors possibles pour l'évolution de ce genre de système (14). Mais, le chaos apparaît aussi dans des systèmes conservatifs, c'cst-à-dire qui conservent leur énergie (15). Notons enfin que nous avons ici évoqués essentiellement le chaos résultant d'une évolution purement temporelle. Il existe également un chaos qui passe aussi par les changements de structures physiques et donc géométriques du système (16). C'est ce qui se produit dans l'écoulement d'un liquide quand s'installe la turbulence.

Finalement, la théorie des systèmes dynamiques témoigne de la richesse des comportements possibles d'un même système selon les contraintes de ses variables caractéristiques, notamment des conditions initiales, et de son environnement.

Le devenir d'un tel système, même s'il aboutit au chaos, est entièrement déterminé par les équations le gouvernant. Ce devenir est certes irréversible. Mais, si les conditions de l'environnement changent à nouveau, il peut changer du tout au tout. Si l'amplificateur siffle parce qu'il se met à osciller, l'arrêt de l'alimentation électrique $\mathrm{y}$ met un terme. Le chaos peut conduire à la destruction du système, mais il est important de souligner qu'il peut aussi être l'occasion pour des nouvelles structures d'apparaître, comme le prévoit la thermodynamique des processus irréversibles. Finalement, tout système physique possède des plages de fonctionnement régulières, mais il en a aussi d'autres qui sont erratiques ou chaotiques. Le passage d'un régime régulier à un régime chaotique dépend des valeurs prises d'une part par les variables du système et d'autre part par les paramètres caractérisant l'influence de l'environnement.

(14) Cf. Manneville, «Structures dissipatives et turbulence », p. 375 s., 380 s. et 396 ; Bergé, Pomeau, Vidal, L'ordre dans le chaos, p. 113-116; Lesne, Méthodes de renormalisation, p. 170-187.

(15) C'est le théorème de Kolmogorov-Arnold-Moser, cf. Lesne, Méthodes de renormalisation, p. 187-195. Certains auteurs écartent cette possibilité, ainsi Bergé, Pomeau, Vidal, $L$ 'ordre dans le chaos, p. 16-24. Mais il faut dire que le chaos résulte davantage du mélange dans un volume de l'espace des phases que de sa diminution. 1998.

(16) Cf. P. Bergé, Y. Pomeau, C. Vidal, L'espace chaotique, Paris, Hermann, 


\section{Le chaos dans les textes bibliques}

Plusieurs textes bibliques évoquent le chaos sans qu'il soit désigné par un mot précis. Le terme de chaos est employé ici pour parler d'une réalité complexe désignée dans la Bible par tout un ensemble de vocables. C'est le cas de Gn 1, 2 qui en constitue un bon exemple. On parle de tohû wabohî, réalité informe et sans vie possible. Le terme hébreu tohû renvoie à Tyamat, un monstre des mythes de création babyloniens. De fait le complexe qu'est le chaos est constitué non seulement par les monstres mythiques : Léviathan, Rahab, le dragon, le serpent, mais aussi d'autres éléments mythiques caractérisant les origines, comme les ténèbres, l'abîme et les eaux.

Deux remarques sont nécessaires pour comprendre la notion de chaos dans les textes bibiques qui en parlent. Premièrement, le chaos est toujours désigné à l'aide d'éléments mythiques. Il est légitime de s'interroger sur le statut des textes comprenant des éléments mythiques. On pourrait dire qu'il s'agit seulement, dans ce genre de textes, d'images telles que les poètes en emploient pour frapper. Comme de dire, aussi, que ces textes, provenant, par voie d'emprunt, de mythologies non bibliques, perdent pour cette raison toute valeur de message et sont ramenés à l'état de résidu qui pourrait disparaître sans que la Bible et nous-mêmes y perdent. Mais la Bible est allée plonger... jusque dans les grands fonds des croyances païennes. C'est, chaque fois, pour délivrer et accompagner jusqu'à nous la parole qui s'y cache et qui est destinée à tous les hommes (17). On voit par conséquent qu'on ne peut, pour des raisons méthodologiques, écarter les textes bibliques contenant des ćlćments mythiques en voyant seulement dans cette manière de faire un effet poétique ou un vestige archaïque figurant dans la Bible et qui n'a plus lieu d'être. Les mythes exposent la nature des choses. Il s'agit d'exprimer d'une certaine manière quelque chose d'important concernant le créé qui reste cependant, au moins en partie, inexplicable. Ces passages contribuent ainsi à la révélation concernant le créé.

Une deuxième remarque confirme l'importance des passages qui mentionnent tout ou partie des " éléments " constituant le chaos. Ils appartiennent à tous les genres littéraires de la Bible : récits narratifs (Gn 1) ou apocalyptiques (Is 24-27), textes liturgiques (Ps 74, 77), proclamations prophétiques (Is 51 ), réflexions sapientielles ( $\mathrm{Jb} 38$ 40). Il s'ensuit que les textes parlant du chaos figurent dans toutes les traditions théologiques qui ont contribué à l'écriture de la Bible. Il y a bien entendu des différences entre ces traditions au sujet de la

(17) P. Beauchamp, Psaumes. Nuit et jour, Paris, Seuil, 1980, p. 201. 
place qu'elles donnent au chaos, mais le fait demeure que le chaos est intégré par toutes les tendances du judaïsme. C'est pourquoi, pour une première analyse, on peut s'en tenir à une approche globale, ce que nous faisons dans la suite de cette étude.

Le chaos existe donc dans la Bible, ou tout au moins un ensemble de choses que l'on peut regrouper sous cette appellation. Quelle en est la signification?

Dans la Bible le chaos apparaît comme ce qui est sans ordre, sans forme et sans vie. Il est, pourrait-on dire, une non-vie dont une image est le désert. Mais, il est actif et s'oppose par un travail de sape à tout ce qui est organisé et structuré. Le chaos doit par conséquent être contenu et seul le Créateur le peut (Ps 24, 2). La Bible utilise une autre image pour décrire l'omniprésence du chaos, celle des rives et du rivage qui limitent les eaux. De même que l'eau imprègne la terre à partir des lieux qu'elle occupe, de même le chaos imprègne le créé. Comme l'eau use et détruit peu à peu, de même le chaos use toute chose et toute vie jusqu'à les détruire. Au chaos sont ainsi associés la mort et le shéol (Jon 2, 3-6). La tradition ultérieure du judaïsme du second Temple parlera des lieux "sous terre", des enfers, pour exprimer cette réalité.

C'est pourquoi l'ordre naturel, les choses, les êtres vivants peuvent à tout moment être remis en question si Dieu laisse faire le chaos. Deux exemples : le déluge et la traversée de la mer rouge. Le déluge est en effet compris comme étant dû aux eaux de l'Abîme (Gn 7, 11 et 8,2). De même, la traversée de la mer rouge et l'engloutissement des Égyptiens sont considérés comme ayant engagé les eaux de l'Abîme (Ex 15, 5).

Cette brève description révèle que le chaos n'est pas un élément de la création comme les autres réalités qui la composent. Celles-ci sont localisées et nommées. Elles sont en relation les unes avec les autres, chacune à sa place. Le chaos ne peut être considéré comme tel. Il n'est pas simplement l'ensemble des puissances déso,ganisatrices que l'on peut localiser et nommer, comme les tremblements de terre, les tempêtes, les épidémies, les famines, etc. Il constitue aussi une réalité universelle, impossible à localiser, insaisissable et impossible à nommer. Le chaos est sous-jacent à tout le créé et c'est en le réduisant et en le limitant que Dieu fait exister le monde et l'être humain et qu'il les mène à leur accomplissement sous l'impulsion du Logos et du Pneuma divins (18).

Le chaos n'est donc pas une propriété naturelle et l'organisation du créé doit être garantie par la puissance divine. Deux traits le

(18) Voir A. Ganoczy, p. 117-118. 
confirment. D'une part, le créé n'existe que parce qu'il est nommé par Dieu, d'où le thème biblique de la création par la parole (19). D'autre part, si le chaos fait partie du processus créateur, il disparaît à l'achèvement de la création (Ap 20,1). Mais, si le chaos est limité par le Créateur sans être éliminé tout de suite de l'acte créateur, c'est qu'il a sa place dans l'accomplissement du projet divin (20).

L'accomplissement de ce projet constitue le salut. Le créé est alors perçu non seulement comme un donné, mais aussi comme une réalité en devenir où le Créateur poursuit la réalisation de son dessein.

\section{Chaos et devenir du créé}

Dans toute la Bible, et pas simplement dans les deux premiers chapitres de la Genèse, figurent des textes parlant du monde comme création en devenir. Commençons par deux extraits de psaumes. On ne pense pas spontanément aux psaumes pour chercher ce que la révélation biblique veut nous dire du monde et de son devenir. C'est déjà une bonne raison pour y aller voir. Une autre raison, plus importante on s'en doute, est que ces prières expriment la conception du monde et de la vie de tout un chacun en Israël d'avant notre ère.

Un premier texte est révélateur de la manière d'agir de Dieu selon la Bible. Il s'agit du Ps 74, 13-14. C'est une lamentation communautaire. L'auteur fait référence à la destruction du sanctuaire autrefois, à la désolation de la ville sainte par les impies, entendez les nonisraélites (v. 1-11), à laquelle il compare la situation de son époque (v. 18-23). Le psaume demande à Dieu d'intervenir. On pourrait penser qu'il s'agit d'un parallèle avec le retour d'exil à partir de 538 . C'est vrai d'une manière. Nous le verrons plus loin. Mais le psaume renvoie explicitement à la création en reprenant quelques éléments fondamentaux du mythe rapportant la geste créatrice où le Créateur crée en déployant sa force contre les puissances du chaos : Toi, tu as maîtrisé la mer (yâm) par ta force, tu as fracassé les têtes des dragons (tanînîm) sur les eaux, tu as fracassé les têtes de Léviathan (lewyatan) (Ps 74, 13-14).

Les puissances du chaos sont signifiées par la mer et ses monstres, ici les dragons et Léviathan. Il s'agit des eaux primordiales que l'on retrouve dans quasiment tous les mythes de création.

(19) Voir P. Beauchamp, Création et séparation. Étude exégétique du chapitre premier de la Genèse, Paris, DDB, 1969, p. $112 \mathrm{~s}$.

(20) Le chaos est à la fois une réalité universelle sous-jacente au créé et un élément cosmique : Beauchamp, Création et séparation, p. 167. 
Le psaume en appelle également à Dieu qui sépare le jour de la nuit en établissant le soleil et la lune (v. 16). Ainsi le psaume nous parle de la création. Il rappelle la geste divine. Mais est-ce simplement le rappel que le Créateur détient la puissance sur le créé et qu'il peut par conséquent intervenir en faveur du peuple maintenant? C'est l'interprétation courante de ce passage (21).

Pourtant, il nous semble qu'il y a plus qu'un appel à la puissance divine. Dieu peut agir maintenant parce qu'il est le Créateur, il agit en créant. Bien sûr il peut faire du radicalement nouveau, c'est la création ex nihilo. Mais, il crée en permanence en maintenant le créé dans l'existence avec son organisation et en jouant sur elle. Tout acte divin est créateur aussi en ce sens. Et c'est bien le cas ici. Un acte de Dieu en faveur de son peuple touche nécessairement au créé. La confirmation de cette idée se trouve dans d'autres textes comme le Ps 77 par exemple. Ce psaume est aussi une lamentation, mais individuelle cette fois-ci. Le psalmiste, dans une situation de détresse, demande à Dieu d'intervenir, comme dans le Ps 74, et de la même manière il fonde sa demande sur l'intervention par excellence de Yahvé en faveur de son peuple Israël : la libération d'Égypte, le passage de la mer rouge, l'exode, actions présentées comme des actes de création identiques à ceux du commencement (v. 12-21), comme on l'avait vu plus haut.

L'exode compris comme acte créateur se retrouve ailleurs dans la Bible. Ainsi, dans un passage du Second Isaïe rédigé à la fin de l'exil, Yahvé conduit son peuple en triomphant des puissances. Nous retrouvons le cortège déjà rencontré : Rahab, le dragon (tanîn), la mer (yâm) et l'abîme (tehôm). Ces deux derniers éléments figurent aussi dans le récit de création de Gn 1,2. Pour le prophète, le retour d'exil en terre promise est analogue à la sortie d'Égypte et à l'action de Yahvé à cette occasion :

Surgis, surgis, revêts-toi de puissance, bras de Yahvé [...] N'est-ce pas toi qui as taillé en pièces Rahab, transpercé le dragon? N'est-ce pas toi qui as dévasté la mer, les eaux de l'abîme gigantesque, qui as fait du fond de la mer un chemin pour que passent les rachetés?

Les affranchis de Yahvé reviendront, ils entreront dans Sion au milieu des acclamations (Is 51, 9-11).

Bien plus encore, l'exode compris comme création nouvelle devient même le paradigme de l'agir salvifique de Dieu. Ainsi en Sg 19 tous les éléments du créé contribuent à la libération des Israë-

(21) Voir les références dans R.E. Brown, J.A. Fitzmyer, R.E. Murphy, The New Jerome Biblical Commentary, London, Geoffrey Chapman, 19902, p. 538, commentaire qui suit cette interprétation. 
lites par le passage de la mer rouge et à la victoire sur les Égyptiens non seulement par leur engloutissement dans les eaux de l'abîme, mais aussi par les plaies d'Égypte comprises comme actes de création.

Un dernier passage, bien après l'exil, mais inséré dans le livre d'Isaïe (Is 24-27), reprend l'idée que le rétablissement d'Israël sur sa terre passe par la victoire de Yahvé sur les puissances : l'abîme, le dragon, Léviathan $(27,1)$, mais aussi sur les puissances célestes $(24,21)(22)$. On voit bien que l'univers tout entier est concerné par l'action de Dieu sur le chaos. Dans cette perspective, dans le devenir du monde et des hommes interviennent non seulement les causes naturelles et l'action humaine, mais aussi les puissances du chaos, l'ensemble étant entre les mains de Dieu.

En conclusion, les notions scientifique et biblique de chaos ne désignent pas totalement la même chose. Conséquemment le chaos n'a pas le même statut. Pour la science le chaos est un mode de fonctionnement possible pour tout système. Il est entièrement « naturel ». Le chaos biblique quant à lui est ambivalent. D'une part il se manifeste dans le créé par tous les phénomènes perturbant l'ordre courant des choses. En ce sens le chaos appartient au créé et rejoint ce que décrit la science. D'autre part il constitue une réalité sousjacente au créé en tant qu'univers organisé, et en ce sens il n'appartient pas au créé, mais il le traverse de part en part comme puissance désorganisatrice contenue et utilisée par le Créateur.

Mais, du fait du statut ambivalent du chaos dans la Bible, les conceptions scientifique et biblique du chaos peuvent être articulées l'une par rapport à l'autre. Les processus de désorganisation et de chaos mis en évidence par la science sont signes d'une réalité plus profonde qui n'est pas accessible telle quelle, mais que, selon la Bible, le Créateur utilise pour faire advenir la création nouvelle. Le chaos est alors un élément de transformation positive. Pourtant, au chaos biblique appartiennent aussi souffrance et mort. Cela conduit à s'interroger d'une part sur leur place par rapport à l'accomplissement du dessein de Dieu et d'autre part sur l'attitude de l'être humain par rapport au chaos biblique. L'analyse de la notion de chaos ouvre ainsi des perspectives à la théologie qui demandent d'être développées pour elles-mêmes. Ce ne peut être l'objet que d'une autre étude.

Jacques FANTINO

Université de METZ

(22) Is 24, 21-27, 2 constitue une section; voir The New Jerome Biblical Commentary, p. 244-245. 